\title{
Use of a comprehensive patient safety tool in primary care practices
}

\author{
Joanne M. Pohl, PhD, ANP-BC, FAAN, FAANP (Professor) ${ }^{1}$, Radhika Nath, PhD (Formerly Senior Research \\ Scientist) ${ }^{2}$, Kai Zheng, PhD (Associate Professor) ${ }^{3}$, Fred Rachman, MD (Chief Executive Officer) ${ }^{4}$, David N. Gans, \\ MHA (Senior Fellow Industry Affairs) ${ }^{5}$, \& Clare Tanner, PhD (Program Director) ${ }^{6}$ \\ ${ }^{1}$ Emerita, The University of Michigan School of Nursing, Ann Arbor, Michigan \\ ${ }_{2}^{2}$ Medical Group Management Association, Englewood, Colorado \\ ${ }^{3}$ School of Public Health, School of Information, and School of Nursing, The University of Michigan, Ann Arbor, Michigan \\ ${ }^{4}$ Alliance of Chicago Community Health Services, Chicago, Illinois \\ ${ }^{5}$ MGMA-ACMPE, Englewood, Colorado \\ ${ }^{6}$ Center for Data Management and Translational Research, Michigan Public Health Institute, Okemos, Michigan
}

\section{Keywords}

Safety; primary care; measurement; work environments.

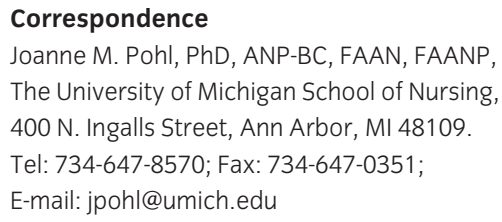

doi: $10.1111 / 1745-7599.12021$

\begin{abstract}
Purpose: To present a tool that can be used to evaluate patient safety in both nurse-led and physician-led practices.

Data source: This article describes our experience with the Physician Practice Patient Safety Assessment (PPPSA) tool in six safety net practices-three of which were primary care nurse-managed health centers and three were physician-led federally qualified health centers. The information provided is from the tool itself and how it might be used in clinical settings, especially primary care.

Conclusions: The PPPSA is a tool to measure the extent to which patient safety practices are rigorously and systematically implemented throughout a health center. The tool's methodology requires discussion and consensus, incorporating a team approach with multiple perspectives within a center. It is designed to promote changes in practices that would improve patient safety. Implications for practice: The tool has enormous relevance for primary care settings, especially those preparing themselves for patient-centered medical home status and meaningful use. But most important, it has relevance as we create healthcare environments that promote patient safety and a practice culture that is truly patient centered.
\end{abstract}

Following two milestone reports published by the Institute of Medicine (IOM, 2000, 2001) preventable medical errors have received much attention in the United States. In addition, patient-centered care and the patientcentered medical home (PCMH) along with meaningful use through the use of electronic health records (EHRs) have gained prominence in addressing patient safety and healthy practice environments. However, assessing and measuring patient safety in primary care practices can be challenging. As part of a larger health information technology research grant (Dennehy et al., 2011; Zeng et al., 2009), that addressed the objectives of patient safety and quality outcomes through use of EHRs among safety net practices in primary care (Dennehy et al., 2011; Zeng et al., 2009), the research team wanted to identify a tool that would be both clinically and organizationally useful as well as meet our research objectives.

This article presents the use of the Physician Practice Patient Safety Assessment (PPPSA; Institute for Safe Medication Practices, Health Research and Educational Trust and Medical Group Management Association, 2006), which addresses the broad range of patient safety issues including organizational climate and structure. Although the tool title is physician centric, we found it to be valuable to any primary care practice, whether led by physicians or nurse practitioners (NPs). The PPPSA was administered in six safety net primary care settings. Three of these were nurse-managed health centers (NMHCs), where the PPPSA was administered before and after EHR implementation. The other three were federally qualified 
health centers (FQHCs), where the instrument was administered post-EHR implementation as their go-live date had occurred prestudy. In this brief article, the PPPSA is described, as well as its methodology for use, as a clinical resources tool that addresses broad patient safety issues. The process in using the tool is a key strength sometimes overlooked in favor of the results of the tool. We describe the assessment process and lessons learned that could be applied to other primary care practices. Data from the tool are presented in another paper in process. Study protocols were approved by Institutional Review Boards with Federal Wide Assurance at Michigan Public Health Institute, University of Michigan, and relevant clinical sites.

\section{PPPSA tool}

The PPPSA was originally developed through a grant from the Commonwealth Fund by the Medical Group Management Association (MGMA) in partnership with the Institute for Safe Medication Practices (ISMP) and the Health Research and Educational Trust to heighten providers' awareness of safe practice and create a "reference point and baseline" for practices to improve and support patient safety. The PPPSA was created using multipronged approaches such as expert panels, literature reviews, and guidelines from various organizations involved in patient safety issues. Ongoing usage of the instrument has lent it to further analysis in order to validate the instrument as well as to improve it. Practices can choose to submit their data to MGMA's national databank and receive benchmarks. The PPPSA is currently in the public domain and available at http://www.mgma.com/pppsa.

The PPPSA addresses multiple areas related to patient safety. The tool begins with a demographics section that describes the practice type (12 items) and level of information technology adoption (15 items). The remaining parts of the survey contain six domains (see Table 1 for sample items): medications, handoffs and transitions, surgery/anesthesia and sedation/invasive procedures (six items-this domain was not assessed in our study because of its lack of relevance in primary care contexts), personnel qualifications/competency, practice management and culture, and patient education and communication. Within each domain, questionnaire items measure the extent to which a healthcare practice (vs. practitioner) has fully implemented patient safety processes. The response options include $1=$ no activity; $2=$ considered but not implemented; 3 = partially implemented in some areas; $4=$ fully implemented in some areas; $5=$ fully implemented in all areas; and $6=$ not applicable/NA.

Particularly noteworthy is the recommended process for using the tool. Because the tool was designed to be practice-based versus provider-based, the PPPSA should be completed collectively by providers, key managerial, and other staff in a group setting. This unique methodology is one we want to emphasize as it provides an opportunity to foster discussion among management, providers, and other staff and come to agreement/consensus on each item as well as jointly identify issues that might be more challenging or otherwise overlooked. For our grant purposes, practices were asked to choose participants, which included front-line EHR clinician users, office managers, medical assistants, and front desk staff. Results were shared with each practice at site visits, consistent with how the data were initially collected allowing for more discussion. Because of variations in the go-live of the EHR at the sites, we were able to compare pre- and postimplementation surveys in only the three NMHCs.

\section{PPPSA process}

For our research purposes, interviews using the tool were led by a skilled interviewer from the research team reading the question to the group with the group coming to consensus on the response for each item. Each participant had a hard copy of the PPPSA during the interview. However, the PPPSA was designed for clinical use and to be practice-led. That is, a skilled interviewer is not necessary to lead the assessment and discussion for nonresearch purposes. The process of discussion leading toward consensus and feedback proved to be highly productive in terms of identifying areas of strengths and areas that needed more attention. The interviews ranged from 1 to $2 \frac{1}{2} \mathrm{~h}$. Centers were provided with results of the PPPSA, both in terms of a written report (with benchmarks) and during debriefings between site leaders and the research team. Where applicable, notes taken during these debriefings were used to interpret data in the discussion. Although the tool is titled "Physician Practice Patient Safety Assessment," we did change terminology to "clinician" because there were other providers in addition to physicians, such as NPs and physician assistants (PAs).

The process of the interviews in which all attendees at each site needed to come to consensus on each item was quite powerful. With some items there would be immediate agreement, while on others, one person might place the response value at " 5 ," "fully implemented" and someone else might say "do you really think we have fully implemented that"? Then there would be in-depth conversation in terms of interpretation and moving to agreement. The discussions and discrepancies were site-specific and found to be most poignant around patient safety issues and where a practice as a whole may need to change 
Table 1 PPPSA domains and sample questions

\begin{tabular}{|c|c|c|}
\hline PPPSA domains & Number of items & Sample questions from PPPSA tool \\
\hline Medications & 17 & $\begin{array}{l}\text { - A complete medication history including OTC medication is obtained at each visit } \\
\text { - All prescriptions are entered into an office-based electronic prescribing system } \\
\text { - Patients are provided with up-to-date list of all medications they are receiving upon leaving the visit }\end{array}$ \\
\hline Handoffs and transitions & 11 & $\begin{array}{l}\text { - When the practice transfers responsibility for the care of a patient to another provider, practice, or } \\
\text { institution, the practice identifies the clinician responsible for accepting the patient and confirms } \\
\text { that the clinician receives and accepts responsibility for the patient. } \\
\text { - The practice maintains a process to communicate all medications that a patient is receiving when } \\
\text { admitted to a hospital, nursing home, home care agency, rehabilitation center, etc. } \\
\text { - The results of laboratory, pathology, and imaging tests are communicated to the patient in a timely } \\
\text { manner ( } 24-48 \text { h), and the practice confirms and documents that the patient received the results. }\end{array}$ \\
\hline $\begin{array}{l}\text { Personnel/Qualifications/ } \\
\text { Competency }\end{array}$ & 10 & $\begin{array}{l}\text { - The practice maintains a system to periodically assess nursing and support staff competency that } \\
\text { is appropriate for the services and procedures they perform } \\
\text { - The practice maintains a system to periodically assess physician competency that is appropriate } \\
\text { for the services and procedures they perform } \\
\text { - All new physicians, NPs, and PAs receive a structured orientation to the practice's policies, } \\
\text { protocols, procedures, administrative guidelines, etc. }\end{array}$ \\
\hline $\begin{array}{l}\text { Practice } \\
\text { management/Culture }\end{array}$ & 22 & $\begin{array}{l}\text { - A system for reporting errors, such as incident reports, is in place and is supported by a culture of } \\
\text { - A prety that allows for open collection and sharing of the data within the practice. } \\
\text { staff, routinely followed and supported by a culture of safety that allows for open collection and } \\
\text { sharing of the data within the practice. } \\
\text { - When errors or near misses occur, educational efforts are widespread among all clinicians and } \\
\text { nonclinical personnel who may make a similar error, rather than remedial and directed only at } \\
\text { those who were involved in the error. } \\
\text { - Job descriptions for all office personnel include requirements to speak up about safety issues, } \\
\text { change practices to enhance safety, share errors, ask for help when needed, and other elements } \\
\text { of shared accountability for safe practices. }\end{array}$ \\
\hline $\begin{array}{l}\text { Patient educa- } \\
\text { tion/Communication }\end{array}$ & 13 & $\begin{array}{l}\text { - Patients are assessed for their financial and physical ability to obtain prescriptions and medical } \\
\text { - } \text { Information on patients' lifestyle, family, home environment, and work is collected and used to } \\
\text { develop a care plan when appropriate. } \\
\text { - Patients are routinely asked to repeat back what they hear to help the clinician clarify any } \\
\text { instructions. } \\
\text { - Patient participation is actively sought in decisions regarding their own care. } \\
\text { - } \text { A process is in place to insure timely follow-up of e-mail and telephone correspondence with } \\
\text { patients and other healthcare providers. }\end{array}$ \\
\hline
\end{tabular}

Reprinted with permission from MGMA-ACMPE, 104 Inverness Terrace East, Englewood, Colorado 80112. www.mgma.com. Copyright 2006-2009.

or realize that they were actually on track but some staff and clinicians were not aware of certain practice policies or expectations. All five PPPSA domains had quite a range of responses across the six practices. Some practices had more policies in place than others. But overall-and the key point we want to make-the tool provided essential discussion around patient safety and practice culture issues and in some cases there were elements that practices had not addressed, but the discussion identified those areas and resulted in plans to change or improve a practice. For example, at one site there was extensive discussion about the practice management/culture domain items related to processes in place for reporting errors openly, feeling safe in a practice, and job descriptions including requirements to speak up about safety (see Table 1 with sample items). Staff and clinicians at this particular practice thought they could do much better on some of the items and made plans to address it.

The patient education and communication domain was evaluated quite highly overall at most of the sites. These were all safety net practices, which have a culture of addressing the needs of fairly vulnerable populations, so that many of the items in this section were familiar core values and fully in place. For example, it is not unusual in NMHCs and FQHCs to assess ability to pay for prescriptions and care because the mission of these centers is to address the needs of vulnerable populations. Although most interviewees indicated they were quite satisfied with communication in the practices, others felt it could be stronger. 


\section{Lessons learned and recommendations}

We found that providing the collective process of assessment and feedback on the PPPSA in a group discussion setting provoked essential discussions among the staff and the managerial team at the study sites. The PPPSA tool was found to be most practical for clini$\mathrm{cal} /$ organizational use. Coming to consensus on each item through discussion during the focus group type session was critical and gave clinicians and other staff an opportunity to engage in discussions about patient safety and practice culture that might not otherwise occur. We also found that staff and clinicians wanted to talk about these issues and reported not having much opportunity to do so. The PPPSA facilitated that needed discussion. We recommend that the PPPSA be repeated annually to stay on top of patient safety processes acknowledging that new clinicians and staff need to have the discussions that occur with this tool. It would also give the practice a sense of change and improvement or highlight the need for more change in specific areas. The PPPSA could facilitate ongoing discussion to emphasize patient safety, monitor improvement, and focus leadership on the effect of particular investments of attention and resources. We recommend practices compare themselves not only to the previous year's assessment but to other practices, and available national benchmarks. Both NMHCs and FQHCs could be submitting data to MGMA to help create a larger data pool and aggregate data from safety net practices.

The tool was particularly useful for our purposes in looking at patient safety and the impact an EHR might have on medication safety, for example. Based upon our experience, the PPPSA may be a valuable adjunct to other instruments to assess the value and impact of an EHR. The PPPSA is also quite congruent with the objectives of the Centers for Medicaid and Medicare Services' (CMS) EHR Meaningful Use incentive program (Centers for Medicaid and Medicare Services, 2010). Meaningful use criteria are based on improving quality, safety, efficiency, and reducing health disparities; engaging patients and families, improving care coordination, improving public and population health, and ensuring privacy and security of personal health information.

Items in the PPPSA address patient-centered care as well and could be useful to practices as they ready themselves for PCMH accreditation or advancing their PCMH level. For sites implementing an EHR, we would recommend using the PPPSA preimplementation as well as postimplementation. The tool has relevance for all practices, whether they have an EHR or not.

\section{Conclusion}

PPPSA is a technology-agnostic tool to measure the extent to which patient safety practices are rigorously and systematically implemented throughout a health center. The tool requires discussion and consensus, incorporating multiple perspectives within a center. In our study, we used the tool as a component to assess patient safety issues in implementing EHRs in resource-challenged safety net settings such as FQHCs and NMHCs. Compared with other private practices, these safety net practices address the needs of patients with less education and income, representing a very diverse payer mix, along with significant chronic and mental health needs. The complex needs in these settings can present challenges in emphasizing patient safety. Using the PPPSA, with its methodology of group discussion and consensus provided a useful process for addressing unique patient safety issues in these settings.

\section{Acknowledgments}

This work was funded by the Agency for Healthcare Research and Quality (R18 R18HS017191-01) "A Partnership for Clinician EHR Use and Quality of Care" (PI: J. M. Pohl). The authors wish to acknowledge the other members of the research team, Erin Kaleba, Andrew Hamilton, Joanne White, and Mike Soltis.

\section{References}

Centers for Medicaid and Medicare Services. (2010). Retrieved from https://www.cms.gov/Regulations-and-Guidance/Legislation/ EHRIncentivePrograms/downloads/MU_Stagel_ReqOverview.pdf

Dennehy, P., White, M., Hamilton, A., Pohl, J. M., Tanner, C., Onifade, T. J., \& Zheng, K. (2011). A partnership model for implementing electronic health records in resource-limited primary care settings: Experiences from two nurse managed health centers. Journal of the American Medical Informatics Association, 18, 820-826.

Institute for Safe Medication Practices, Health Research and Educational Trust and Medical Group Management Association. (2006). The Physician Practice Safety Assessment ${ }^{\mathrm{TM}}$, Englewood, CO: Medical Group Management Association. Retrieved from http://www.mgma.com/pppsatermsofuse Institute of Medicine. (2000). To err is human: Building a safer health system. Washington, DC: National Academy Press.

Institute of Medicine. (2001). Crossing the quality chasm: A new health system for the $21^{\text {st }}$ century. Washington, DC: National Academy Press.

Zheng, K., McGrath, D., Hamilton, A., Tanner, C., White, M., \& Pohl, J. M. (2009). Assessing organizational readiness for adopting electronic health record systems: A case study in ambulatory practices. Journal of Decision Systems, 8, 117-140. 